In der Arbeit von Jakob Lund über

\title{
Berichtigung.
}

\section{Die Beziehungen zwischen den Fettkonstanten}

(Diese Zeitschrift 1922, 44, 113-187)

sind nachstehende Berichtigungen vorzunehmen:

S. 134 in Zeile 1 von oben lies $\mathbf{0 , 0 0 0 2 2 6} \mathbf{N}$ statt 0,00026 .

$\because 138, \pi 7$ von unten lies 0,000026 statt 0,00026 .

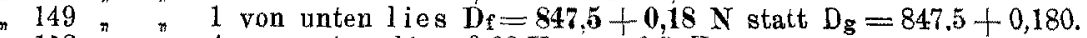

, $153, \pi 4$ von unten, lies $0,08 \mathrm{~V}$ statt $0,8 \mathrm{~V}$.

"154 in der letzten Spalte der Tabelle lies $0,080,0,081$ usw. statt $0,80,0,81$ usw.

" 167 lies viermal $0,08 \mathrm{~V}$ statt $0,8 \mathrm{~V}$.

" $169 \mathrm{im}$ Kopfie der ersten und dritten Tabelle lies $0,08 \mathrm{~V}$ statt $0,8 \mathrm{~V}$.

- 170 lies zweimal $0,08 \mathrm{~V}$ statt $0,8 \mathrm{~V}$.

" 171 lies zweimal $0,08 \mathrm{~V}$ statt $0,8 \mathrm{~V}$.

172 in der letzten Gleichung fur die Glyceride $C_{18}$ lies 89,7 statt 98,7

174 in der 2. Formel lies $0,08 \mathrm{~V}$ statt $0,8 \mathrm{~V}$.

174 in der 4., 6. und 8. Formel lies $0,08 \mathrm{~V}$ statt $0,8 \mathrm{~N}$,

, 174 in der Zeile nach der 6 . Formel lies $\mathrm{g}=\mathbf{0 . 6 8}$ statt $\mathrm{g}=0,50$.

; 174 in der 7. Formel lies 0,68 [a]D statt 0,50 [a]D.

. 175 in der lezten Formel lies $0,08 \mathrm{~V}$ statt $0,8 \mathrm{~V}$.

" 178 in der letaten Formel lies - c. V. statt + c. V.

$" 178$ in Zeile 7 von oben lies $g=$ etwa 0,68 statt $\mathrm{g}=$ etwa 0,50 .

J. Lund.

\section{Beferate.}

\section{Allgemeine analytische Methoden und Apparate.}

H. Lührig: Uber Mikrostickstoffbestimmungen. (Pharm. Zentralhalle 1921, 62, 437-444.) - Verf. hat eine Anzahl Versuche mit dem von Bang beschriebenen Mikro-Destillationsapparat (Verlag von J. F. Bergmann, Wiesbaden) ausgeführt und dabei festgestellt, daß mit dem Verfahren sehr gute Ergebnisse unter Ersparung von Zeit und Material erzielt werden. Zur Bestimmung des Stickstoffes genügen $10 \mathrm{ccm}$ der Lösung, zur Bestimmung des Stickstoffes in organischen Stoffen nach Kjeldahl 0,1 g Substanz and darunter; die Destillation ist in zwei Minuten beendet. Bei Mengen unter $1 \mathrm{mg}$ Ammoniak ist das jodometrische Verfahren mit Verwendung von $1 / 100$ oder $1 / 200$ N.-Säure, von der jeder Kubikzentimeter 0,14 bezw. $0,07 \mathrm{mg}$ Stickstoff entspricht, vorzuziehen. Handelt es sich um Mengen erheblich unter $0,1 \mathrm{mg}$ oder um Spuren Ammoniak, so empfiehlt es sich, auf das kolorimetrische Verfahren zurückzugreifen. Die Mikrobestimmung bewährte sich auch bei der Untersuchung von Abwässern zur Bestimmung des Gesamt Stickstoffs nach Kjeldabl, jedoch, muß dabei zum Übertreiben des Ammoniaks Natronlauge verwendet werden und nicht durch Kochen von Ammoniak befreites Magnesiumoxyd, weil in diesem Falle größere Unterschiede gegenüber der Bestimmung nach dem Makroverfahren eintreten. Bei der Bestimmung des Salpeterstickstoffs nach dem Verfahren von Ulseh zeigte es sich, daß der nach Zusatz von Natronlauge im Destillierkolben gebildete Eisenhydroxydniederschlag das Ammoniak hartnäckig zurückhält, besonders wenn größere Mengen vorbanden sind; es ist daher nötig, in solchen Fällen die Salpeterbestimmung in so grosser Verdünnung vorzunehmen, dab man nur wenige Mílligramm Ammoniak erhält. Lebhafte Siedebewegung und 10 Minuten Destillationsdauer liefern genügend genaue Werte und $0,5 \mathrm{~g}$ Eisen und $1,5 \mathrm{ccm}$ Schwefelsäure genügen völlig bei 3-4 Minuten Reduktionsdauer. 